\title{
Efecto de tres modos ventilatorios como único soporte en un modelo experimental de inflamación sistémica por lipopolisacárido sobre la hemodinamia, fisiología pulmonar e histología
}

\author{
Effect of three ventilation modes as the only support in \\ a pig model with LPS-induced systemic inflammation on \\ pulmonary physiology, histology and hemodynamics
}

Axel E. Guzmán-Cedillo, J. Raúl Olmos-Zúñiga,* Rogelio Jasso-Victoria,* Rogelio García-Torrentera,*

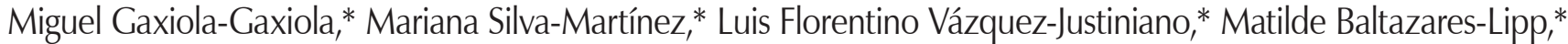
Claudia Hernández-Jiménez,* Edgar Zenteno-Galindo, Felipe Vadillo-Ortega,\$, Ramón González-Camarenall

*Instituto Nacional de Enfermedades Respiratorias Ismael Cosío Villegas, Ciudad de México. ${ }^{\ddagger}$ Universidad

Nacional Autónoma de México, Ciudad de México. §Instituto Nacional de Medicina Genómica,

Ciudad de México. "Universidad Autónoma Metropolitana Iztapalapa, Ciudad de México.

RESUMEN. Introducción: La asistencia mecánica ventilatoria (AMV) en modo liberación por presión de la vía aérea (APRV) y ventilación de alta frecuencia oscilatoria (VAFO) favorecen la protección pulmonar, pero no se utilizan en pacientes con sepsis. Objetivo: Comparar el efecto de la AMV en modo de ventilación controlada por presión (VCP), APRV y VAFO como única terapia sobre la hemodinamia, gasometría, mecánica e histología pulmonar en un modelo porcino de inflamación sistémica inducida por lipopolisacárido de Escherichia coli (E. coli). Material y métodos: Dieciocho cerdos con inflamación sistémica lipopolisacárido
ABSTRACT. Introduction: The airway pressure release ventilation (APRV) and high frequency oscillatory ventilation (HFOV) modes provides lung protection, but is not used in patients with sepsis. Objective: Compared the effect of mechanical ventilation support (MVS) in PCV, APRV and HFOV modes as the only therapy in a pig model of systemic inflammation induced by Escherichia coli lipopolysaccharide on hemodynamics, gasometry, mechanics and lung histology. Material and methods: Eighteen pigs with systemic inflammation were treated only with MVS: group I: PCV, group II: APRV and group III: HFOV.

\section{Abreviaturas:}

AMV = Asistencia mecánica ventilatoria.

APRV = Liberación por presión de la vía aérea.

VAFO $=$ Ventilación de alta frecuencia oscilatoria

LPS = Lipopolisacárido.

$\mathrm{LPA}=$ Lesión pulmonar aguda.

SIRA = Síndrome de insuficiencia respiratoria aguda .

$\mathrm{Vt}=$ Volumen tidal.

VILI = Lesión pulmonar inducida por ventilación.

$\mathrm{VCV}=$ Ventilación controlada por volumen.

$\mathrm{VCP}=$ Ventilación controlada por presión.

$\mathrm{FiO}_{2}=$ Fracción espirada de oxígeno.

PEEP = Presión positiva al final de la expiración.

$\mathrm{FC}=$ Frecuencia cardíaca.

$\mathrm{GC}=$ Gasto cardíaco.

PAM $=$ Presión arterial media.

PMAP $=$ Presión media de la arteria pulmonar.

POAP $=$ Presión de oclusión de la arteria pulmonar.

PVC $=$ Presión venosa central.

$\mathrm{RVP}=$ Resistencias vasculares pulmonares
RVS $=$ Resistencias vasculares sistémicas.

$\mathrm{PaCO}_{2}=$ Presión arterial de dióxido de carbono.

$\mathrm{PaO}_{2}=$ Presión arterial de oxígeno

Qt-Qs = Corto circuito.

Paw = Presión media de la vía aérea.

Raw $=$ Resistencia de la vía aérea.

$\mathrm{P}_{\text {pico }}=$ Presión pico.

$C_{d y n}^{\text {pico }}=$ Distensibilidad dinámica.

$\triangle \mathrm{dyn}=$ Delta de presión.

ANDEVA-MR = Análisis de varianza de medidas repetidas.

WOBV $=$ Trabajo respiratorio.

PMN = Polimorfos nucleares.

SDRA $=$ Síndrome de dificultad respiratoria aguda.

Correspondencia:

Dr. Rogelio Jasso-Victoria

Instituto Nacional de Enfermedades Respiratorias

Ismael Cosío Villegas. Ciudad de México, México.

Correo electrónico: rojavi_2000@yahoo.com

Trabajo recibido: 20-IX-2019; aceptado: 29-X-2019. 
Neumol Cir Torax. 2020; 79 (1): 37-49

(LPS) E. coli, se trataron únicamente con AMV: grupo I: VCP, grupo II: APRV y grupo III: VAFO. Durante seis horas se evaluó la hemodinamia, gasometría, mecánica e histología pulmonar. Resultados: Los tres modos ventilatorios alteran la hemodinamia pulmonar, pero mantienen la gasometría y mecánica pulmonar. El modo VAFO mantiene estables los valores hemodinámicos sistémicos, disminuye la hiperlactatemia $(p<$ 0.05 , ANDEVA-MR) y produce menor daño histológico ( $p<0.05$, KruskalWallis). Conclusión: Los modos VCP, APRV y VAFO utilizados como único soporte en las fases iniciales de inflamación sistémica inducida con LPS en cerdos, mantienen los valores gasométricos y ventilatorios dentro de los parámetros normales; pero, el modo VAFO favorece la recuperación de los valores hemodinámicos sistémicos y produce menor daño histológico.

Palabras clave: Inflamación sistémica, lipopolisacárido, ventilación controlada por presión, ventilación con liberación por presión de la vía aérea, ventilación de alta frecuencia oscilatoria.

\section{INTRODUCCIÓN}

La sepsis es una disfunción orgánica que puede evolucionar a choque séptico por el desarrollo de un proceso inflamatorio sistémico en el que se activan diferentes tipos celulares, vías de señalización, citocinas y especies reactivas de oxígeno, lo cual ocasiona, entre otras cosas, hipoxemia por lesión pulmonar aguda (LPA), ${ }^{1-3}$ cambios hemodinámicos, celulares y metabólicos, caracterizados por hipotensión e hiperlactatemia. ${ }^{4,5}$ Si estos no se corrigen con oportunidad pueden progresar a síndrome de insuficiencia respiratoria aguda (SIRA), falla orgánica múltiple y muerte. ${ }^{3}$

El tratamiento de la sepsis y SIRA se realiza con antibióticos, terapia de líquidos y asistencia mecánica ventilatoria (AMV) ${ }^{2,6,7}$ debido a que presentan $\mathrm{PaO}_{2} / \mathrm{FiO}_{2}<300^{8,9}$ y la AMV actúa como terapia de soporte, limita el progreso de la enfermedad y mantiene los parámetros fisiológicos de forma adecuada. Cabe mencionar que la AMV utilizada durante este proceso incluye el uso de medidas de protección pulmonar que manejan volúmenes corrientes (Vt) bajos, evitan presiones y volúmenes altos al final de la inspiración y espiración, además de evitar altas concentraciones de oxígeno para prevenir la lesión pulmonar inducida por ventilación (VILI). Actualmente no se recomienda un modo ventilatorio en específico. ${ }^{10}$

Los modos ventilatorios comúnmente utilizados durante la sepsis y el SIRA son la ventilación controlada por volumen (VCV) y la ventilación controlada por presión (VCP). ${ }^{11}$ No obstante, estudios clínicos y experimentales han demostrado que estos modos ventilatorios pueden dañar la función y la arquitectura pulmonar, así como la hemodinamia. ${ }^{12,13}$ Por tanto, es necesario buscar y adecuar otros modos ventilatorios que eviten esto y no incrementen las lesiones pulmonares ocasionadas por la inflamación sistémica inducida por la sepsis.

El modo por liberación por presión de la vía aérea (APRV) y ventilación de alta frecuencia oscilatoria (VAFO) proveen
Hemodynamic parameters, gasometric values, lung mechanics and histology were assessed for six hours. Results: The three ventilatory modes alter pulmonary hemodynamics, but maintain gasometry and lung mechanics values. The HFOV remain stable systemic hemodynamic values, decreases hyperlactatemia ( $p<0.05$, ANOVA-MR) and produces less histological damage ( $p<0.05$, Kruskal-Wallis). Conclusions: The PCV, APRV and HFOV modes used as the unique support in the initial phases of sepsis induced with LPS of Escherichia coli in pigs, maintain the gasometric and ventilatory values within normal parameters; but, the HFOV mode favors the recovery of systemic hemodynamic values and produces less histological damage.

Keywords: Systemic inflammation, lipopolysaccharide, mechanical ventilation support, airway pressure release ventilation, high frequency oscillatory ventilation.

de protección pulmonar y mejoran la oxigenación, ya que ambos modos promueven el reclutamiento alveolar y minimizan los efectos hemodinámicos de la $\mathrm{AMV}{ }^{14,15}$ debido a que el modo APRV utiliza dos niveles de presión (alto y bajo) y una relación I:E con un tiempo inspiratorio prolongado (tiempo alto) y un espiratorio corto (tiempo bajo), en adición permite respiraciones espontáneas que mejoran la estabilidad pulmonar y reducen la presión pleural por los movimientos voluntarios del diafragma. ${ }^{16,17}$ Mientras que el modo VAFO utiliza Vt bajos, menores al espacio muerto de las vías aéreas $(1-2 \mathrm{~mL} / \mathrm{kg})$ con frecuencias respiratorias altas (3-15 Hz o 180-900 respiraciones por minuto) mayores a las fisiológicas $\left(1 \mathrm{~Hz}=60\right.$ respiraciones por segundo). ${ }^{18-20}$ Con base en lo anterior se puede pensar que el uso de APRV y VAFO como única terapia de soporte en cerdos con inflamación sistémica inducida por lipopolisacárido (LPS) de Escherichia coli (E. coli) limitarán las lesiones en pulmón, los cambios fisiológicos y mantendrán menores daños al compararse con el modo VCP.

El objetivo de este estudio fue comparar el efecto de la AMV en modo VCP, APRV y VAFO como única terapia sobre la hemodinamia, gasometría, mecánica e histología pulmonar en un modelo porcino de inflamación sistémica inducida por LPS E. coli.

\section{MATERIAL Y MÉTODOS}

Se utilizaron 18 cerdos Landrace, sanos, machos de 55-66 días de edad, con un peso entre $20-25 \mathrm{~kg}$. Este protocolo fue revisado y aprobado por el Comité de Bioética del INERICV (número de protocolo: B19-12) y realizado de acuerdo con las Especificaciones Técnicas para el Cuidado y Uso de Animales de Laboratorio de la Norma Oficial Mexicana ${ }^{21}$ y de la Guide for the Care and Use of Laboratory Animals de Estados Unidos. ${ }^{22}$ Todos los animales fueron confinados en jaulas individuales, en las mismas condiciones ambientales previas al experimento y todos recibieron comida y agua ad libitum. 
Los animales fueron sedados con (4 mg/ $\mathrm{kg}, \mathrm{IM}) \mathrm{de}$ tiletamina-zolazepam (Zoletil, Virbac, Carros, Francia) y la anestesia se mantuvo durante todo el estudio con propofol (4 mg/kg, IV) (Recofol, PISA, Guadalajara, México) y fentanilo $(0.1 \mathrm{mg} / \mathrm{kg}$, IV) (Fentanest, Janssen-Cilag, Puebla, México) como analgésico. Se intubaron orotraquealmente (Endotracheal tube, Rush. Kamunting, Malaysia) y se conectaron a un ventilador (AVEA Vyasis Health care Vyasis Respiratory Care Inc., Yorba Linda, California, USA), con el cual se ventilaron en modo controlado por VCV utilizando $\mathrm{Vt}$ de $6-8 \mathrm{~mL} / \mathrm{kg}$ de peso, frecuencia respiratoria de 25 rpm, fracción inspirada de oxígeno $\left(\mathrm{FiO}_{2}\right)$ de $100 \%$, trigger de 2 y presión positiva al final de la expiración (PEEP) de 5 $\mathrm{CmH}_{2} \mathrm{O}$. El modo VCV fue utilizado para obtener los valores de presión pico $\left(\mathrm{P}_{\text {pico }}\right)$ y presión meseta $\left(\mathrm{P}_{\text {plat }}\right)$ y establecer la presión máxima al iniciar el modo APRV, así como para el registro de los valores basales hemodinámicos, gasométricos y temperatura, los cuales se obtuvieron 30 minutos después de que se observó estabilidad de los mismos en todos los animales.

Mediante arterio- y venodisección se colocaron catéteres vasculares en la arteria carótida (Cordis Corp, Johnson \& Johnson, CA, USA) y vena yugular derecha (Swan Ganz 5 $\mathrm{Fr}$, Edwards Lifesciences, Irvine Cal, USA), a través de los cuales se registraron los parámetros hemodinámicos sistémicos, pulmonares, gasto cardíaco y se realizaron toma de muestras sanguíneas.

Después del registro de los parámetros basales, a través del puerto distal del catéter colocado en la vena yugular, se infundieron $1.5 \mathrm{mg} / \mathrm{kg}$ de LPS E. coli cepa 0111: B4 (Sigma-Aldrich, Suiza) ${ }^{23}$ diluido en $20 \mathrm{~mL}$ de solución salina (2 $\mathrm{mL} / \mathrm{min})$.

La AMV con los diferentes modos ventilatorios se inició inmediatamente después de que se presentó hipotensión severa $(<60 \mathrm{mmHg}$ de MAP) posaplicación del LPS E. coli. Posterior a esto, los animales fueron divididos aleatoriamente en tres grupos de estudio de acuerdo al modo ventilatorio a utilizar como terapia única.

Grupo I $(n=6)$ : inflamación sistémica inducida por LPS E. coli tratada con AMV en modo VCP, utilizando Vt de $6 \mathrm{~mL} / \mathrm{kg}$ para mantener una presión plateau $\left(\mathrm{P}_{\text {plat }}\right) \leq 30$ $\mathrm{CmH}_{2} \mathrm{O}$, PEEP $\left(\leq 5 \mathrm{cmH}_{2} \mathrm{O}\right)$ y las $\mathrm{FiO}_{2}$ se ajustó para mantener $\mathrm{SaO}_{2}$ superior a $90 \%$.

Grupo II $(n=6)$ : inflamación sistémica inducida por LPS E. coli tratada con AMV en modo APRV utilizando presión alta $\left(\mathrm{P}_{\text {alta }}\right) \leq 30 \mathrm{cmH}_{2} \mathrm{O}$, presión baja $\left(\mathrm{P}_{\text {baja }}\right) 5$ $\mathrm{CmH}_{2} \mathrm{O}$, tiempo alto $\left(\mathrm{T}_{\text {alto }}\right) 4$ segundos y tiempo bajo $\left(\mathrm{T}_{\text {bajo }}\right)$ 0.5-0.8 segundos.

Grupo III $(n=6)$ : inflamación sistémica inducida por LPS E. coli tratada con AMV en modo VAFO utilizando un ventilador Sensor Medic 3100B (CareFusion, USA). El modo ventilatorio se estableció aplicando una presión media de la vía aérea (Paw) de $14 \mathrm{cmH}_{2} \mathrm{O}$, amplitud del diafragma
48-54 $\mathrm{cmH}_{2} \mathrm{O}$, frecuencia de $6 \mathrm{~Hz}$, tiempo inspiratorio constante al $33 \%$ y flujo de $20 \mathrm{~L} / \mathrm{min}$. $\mathrm{La} \mathrm{FiO}_{2}$ se modificó para mantener $\mathrm{SaO}_{2}$ por arriba de $90 \%$.

En los grupos I y III se administró pancuronio (Bromurex, PiSA, Guadalajara, México) y fentanilo cada 40 minutos durante todo el estudio para producir relajación y analgesia. El estudio tuvo una duración de seis horas poscambio del modo ventilatorio.

Se valoró la frecuencia cardíaca (FC), gasto cardíaco (GC), presión arterial media (PAM), presión media de la arteria pulmonar (PMAP), presión de oclusión de la arteria pulmonar (POAP), presión venosa central (PVC), resistencias vasculares pulmonares (RVP) y sistémicas (RVS) con un monitor de signos vitales (Carescape B650, General Electric; Helsinki, Finland). Para las gasometrías se utilizó un gasómetro (AVL Compact 2, Graz, Australia), se evaluó la presión arterial de dióxido de carbono $\left(\mathrm{PaCO}_{2}\right)$, relación $\mathrm{PaO}_{2} / \mathrm{FiO}_{2}$ real y la corregida para la ciudad de México (2200 MSNM), corto circuito (QtQs), glucosa y lactato en sangre arterial y venosa. Estas evaluaciones se realizaron al registro de los parámetros hemodinámicos, pulmonares y gasométricos basales a los 15 minutos posteriores a la infusión del LPS E. coli, y cada 30 minutos después de la colocación de los catéteres hasta el final del estudio.

La evaluación de la mecánica pulmonar sólo se realizó en los grupos I y II, debido a que el ventilador de alta frecuencia no registra estos parámetros. Se evaluó la presión media de la vía aérea (Paw), resistencias de la vía aérea $\left(\right.$ Raw), presión pico $\left(\mathrm{P}_{\text {pico }}\right)$, distensibilidad dinámica $\left(\mathrm{C}_{\mathrm{dyn}}\right)$, delta de presión $(\triangle \mathrm{P})$ en el grupo de VCP por medio de: $\mathrm{P}_{\text {pico }}$-PEEP; mientras para APRV: $\mathrm{P}_{\text {alta }}-\mathrm{P}_{\text {baja }}$. También se valoró el trabajo respiratorio (WOBv).

La evaluación radiológica se realizó por medio de rayos $X$ ventrodorsales previo a la infusión del LPS E. coli y a las dos, cuatro y seis horas posterior a haber iniciado los diferentes modos ventilatorios.

La evaluación microscópica se realizó al final del tiempo de estudio (seis horas), se tomaron biopsias del lóbulo pulmonar caudal derecho por medio de esternotomía, se fijaron en formaldehído al 10\% por 24 horas, se incluyeron en parafina, cortados a 4 micras y teñidos con hematoxilinaeosina. Después con microscopia de luz se evaluó el grado de edema, hemorragia, congestión, colapso e inflamación y tipo de células inflamatorias presentes mediante una escala descrita por Koksel y colegas, ${ }^{24}$ la cual describe la severidad de los daños en cuatro grados: grado $1=$ ausente, grado 2 $=$ leve, grado $3=$ moderado y grado $4=$ severo. $^{24}$

Una vez tomadas las biopsias los animales fueron sometidos a eutanasia con sobredosis de pentobarbital sódico $(80 \mathrm{mg} / \mathrm{kg} /$ IV) (Pisabental, PiSA, Guadalajara, México). Cabe mencionar que utilizamos muestras de tejido sano de nuestro banco de tejidos para compararlos con los pulmones lesionados. 


\section{Análisis estadístico}

Para las evaluaciones paramétricas se utilizó análisis de varianza de medidas repetidas (ANDEVA-MR) y Bonferroni; mientras que para las no paramétricas se utilizó Kruskal-Wallis. Todos los valores de $p<0.05$ se consideraron como significativos. El análisis estadístico se realizó con el software SPSS versión 18 (SPSS, Inc., Chicago, IL, USA).

\section{RESULTADOS}

Todos los animales desarrollaron inflamación sistémica en promedio a la primera hora de haberse inyectado el LPS E. coli, el cual se caracterizó por taquicardia, disminución del GC, hipotensión sistémica, hipertensión pulmonar (Tabla 1), hiperlactatemia, hipoglucemia, hipertermia, disminución en la $\mathrm{PaO}_{2}$ e incremento en la $\mathrm{PaCO}_{2}$ (Tabla 2). No todos los animales sobrevivieron al tiempo de estudio establecido, debido a que dos animales de los grupos I (VCP) y II (APRV), así como uno del grupo III (VAFO) fallecieron a las cinco horas posaplicación del LPS E. coli.

\section{Hallazgos hemodinámicos}

Posterior a la producción de sepsis, la POAP (Tabla 1), en todos los grupos, se mantuvo dentro de los parámetros de referencia durante todo el estudio.

La FC aumentó durante todo el análisis en los tres grupos al compararse con los valores basales a partir de la primera hora de haber iniciado los diferentes modos ventilatorios, pero este incremento sólo fue significativo $(p<0.05 \mathrm{AN}$ DEVA-MR, Bonferroni) para el grupo I a las cuatro horas de estudio. Al comparar la FC entre grupos no se observaron diferencias estadísticas ( $p=0.37$ ANDEVA-MR).

El GC disminuyó de manera importante en todos los grupos a las tres horas de haber iniciado los diferentes modos ventilatorios al compararse con los valores basales $(p<0.05$ ANDEVA-MR) (Tabla 1) y así se mantuvo durante toda la investigación (Tabla 1); sin embargo, al comparar entre grupos no se observaron cambios significativos ( $p=$ 0.25 ANDEVA-MR) (Tabla 1).

La PAM en todos los grupos de estudio disminuyó de manera importante (al compararse con los valores basales a partir del cambio de modo ventilatorio y así se conser-

Tabla 1: Comportamiento hemodinámico en cerdos con inflamación sistémica inducida por LPS tratados con tres modos ventilatorios.

\begin{tabular}{|c|c|c|c|c|c|c|c|c|c|}
\hline Hemodinamia & Grupo & Basal & $15 \mathrm{~min}$ & 1 hora & 2 horas & 3 horas & 4 horas & 5 horas & 6 horas \\
\hline \multirow{3}{*}{$\begin{array}{l}P_{\text {cuña }} \\
\text { (mmHg) }\end{array}$} & VCP & $9.4 \pm 1.0$ & $7.4 \pm 1.7$ & $7.4 \pm 1.1$ & $7.0 \pm 1.1$ & $8.0 \pm 0.8$ & $8.2 \pm 1.1$ & $8.0 \pm 1.0$ & $9.2 \pm 0.8$ \\
\hline & APRV & $8.8 \pm 1.3$ & $11.2 \pm 0.7$ & $10.8 \pm 1.3$ & $10.6 \pm 1.0$ & $11.4 \pm 1.3$ & $11.0 \pm 0.8$ & $11.0 \pm 0.5$ & $11.0 \pm 0.5$ \\
\hline & VAFO & $7.8 \pm 0.6$ & $8.8 \pm 1.1$ & $9.4 \pm 0.9$ & $11.0 \pm 1.2$ & $10.0 \pm 1.9$ & $14.2 \pm 3.4$ & $9.7 \pm 0.4$ & $11.0 \pm 1.1$ \\
\hline \multirow[t]{3}{*}{$\mathrm{GC} \mathrm{L/min}$} & VCP & $2.6 \pm 0.2$ & $2.6 \pm 0.5$ & $2.8 \pm 0.4$ & $2.4 \pm 0.5$ & $2.2 \pm 0.2$ & $1.8 \pm 0.4$ & $1.4 \pm 0.2^{*}$ & $1.2 \pm 0.2^{*}$ \\
\hline & APRV & $3.8 \pm 0.6$ & $3.6 \pm 0.5$ & $3.4 \pm 0.4$ & $2.2 \pm 0.6$ & $1.8 \pm 0.4^{*}$ & $1.8 \pm 0.4^{*}$ & $1.4 \pm 0.2^{*}$ & $1.4 \pm 0.2^{*}$ \\
\hline & VAFO & $3.4 \pm 0.3$ & $4.02 \pm 0.2$ & $3.72 \pm 0.3$ & $2.72 \pm 0.3$ & $2.0 \pm 0.2^{*}$ & $2.0 \pm 0.2^{*}$ & $2.3 \pm 0.1^{*}$ & $2.2 \pm 0.4$ \\
\hline \multirow{3}{*}{$\begin{array}{l}\text { PAM } \\
(\mathrm{mmHg})\end{array}$} & VCP & $98.3 \pm 4.6$ & $92.8 \pm 2.6^{*}$ & $64.4 \pm 5.5$ & $65.0 \pm 6.9$ & $60.4 \pm 9.3$ & $61.4 \pm 8.8$ & $44.8 \pm 12.3$ & $41.0 \pm 7.4^{*}$ \\
\hline & APRV & $102.8 \pm 5.7$ & $90.2 \pm 10.3^{\star}$ & $54 \pm 5.3^{*}$ & $50.2 \pm 7.6^{*}$ & $48 \pm 4.9^{*}$ & $45.6 \pm 14.4^{*}$ & $35.4 \pm 7.4^{*}$ & $34.6 \pm 6.0^{*}$ \\
\hline & VAFO & $102.2 \pm 5.6$ & $86.8 \pm 4.1^{*}$ & $67.6 \pm 9.5^{\star}$ & $55.4 \pm 10.0^{*}$ & $69.4 \pm 10.2^{*}$ & $72.8 \pm 2.9$ & $76.0 \pm 2.0^{\star \star}$ & $75.6 \pm 7.9^{\star \star}$ \\
\hline \multirow{3}{*}{$\begin{array}{l}\text { PMAP } \\
(\mathrm{mmHg})\end{array}$} & VCP & $17.6 \pm 1.7$ & $26.8 \pm 4.1$ & $29.6 \pm 3.9$ & $33.4 \pm 4.6^{*}$ & $27.6 \pm 5.7$ & $18.6 \pm 3.3^{* \star *}$ & $16.6 \pm 3.9^{* \star \star}$ & $18.3 \pm 5.1^{\star \star \star}$ \\
\hline & APRV & $19.8 \pm 2.9$ & $31.8 \pm 2.3^{*}$ & $35.4 \pm 3.1^{*}$ & $34.6 \pm 4.6^{*}$ & $41.4 \pm 2.7^{\star}$ & $34.0 \pm 5.5^{*}$ & $32.2 \pm 5.0^{*}$ & $29.0 \pm 5.0^{*}$ \\
\hline & VAFO & $20.2 \pm 1.8$ & $29.8 \pm 2.1^{*}$ & $30.0 \pm 2.4^{*}$ & $34.8 \pm 2.6^{*}$ & $35.6 \pm 3.6^{\star}$ & $31.0 \pm 3.0^{*}$ & $37.4 \pm 0.2^{*}$ & $36.4 \pm 3.6^{*}$ \\
\hline \multirow{3}{*}{$\begin{array}{l}\text { RVP (dinas/ } \\
\text { seg/ } / \mathrm{cm}^{5} \text { ) }\end{array}$} & VCP & $289.4 \pm 105.3$ & $701.0 \pm 261.2$ & $731.2 \pm 258.8$ & $1,138.0 \pm 426.9^{*}$ & $791.0 \pm 264.6$ & $944.8 \pm 314.6$ & $1,123.8 \pm 422.1$ & $1,552.0 \pm 442.5$ \\
\hline & APRV & $212.6 \pm 89.0$ & $659.4 \pm 356.0$ & $822.4 \pm 202.3^{*}$ & $902.0 \pm 101.8^{*}$ & $1,025.4 \pm 194.8^{*}$ & $982.2 \pm 158.7^{*}$ & $1,176.4 \pm 209.6^{*}$ & $1,085.6 \pm 193.7^{*}$ \\
\hline & VAFO & $252.6 \pm 43.7$ & $310.6 \pm 47.8$ & $526.6 \pm 97.9$ & $698.6 \pm 90.5$ & $700.2 \pm 120.5^{\star}$ & $937.2 \pm 199.4$ & $1,110.8 \pm 25.3^{*}$ & $1,045.6 \pm 154.1^{*}$ \\
\hline \multirow{3}{*}{$\begin{array}{l}\text { RVS (dinas/ } \\
\mathrm{seg} / \mathrm{cm}^{5} \text { ) }\end{array}$} & VCP & $2,330 \pm 137.6$ & $2,330.8 \pm 137.6$ & $1,732.2 \pm 323.8$ & $1,995.2 \pm 226.7$ & $2,253.8 \pm 410.8$ & $3,014.6 \pm 446.8$ & $2,967.6 \pm 277.9$ & $3,154.8 \pm 370.1$ \\
\hline & APRV & $2,211.8 \pm 254.0$ & $2,688.4 \pm 255.0$ & $1,763.0 \pm 216.6$ & $1,803.8 \pm 219.0$ & $2,013.2 \pm 282.3$ & $2,397.2 \pm 525.4$ & $2,523.6 \pm 434.6$ & $2,540.4 \pm 422.3$ \\
\hline & VAFO & $2,247.8 \pm 330.2$ & $2,247.8 \pm 330.2$ & $1,373.2 \pm 333.5$ & $1,707.2 \pm 450.2$ & $2,074.2 \pm 132.4$ & $2,832.6 \pm 419.5$ & $2,323.8 \pm 68.9$ & $2,070.2 \pm 287.0$ \\
\hline \multirow{3}{*}{$\begin{array}{l}\text { PVC } \\
(\mathrm{mmHg})\end{array}$} & VCP & $6.6 \pm 0.2$ & $7.0 \pm 0.5$ & $7.0 \pm 1.2$ & $8.8 \pm 2.3$ & $4.2 \pm 1.0$ & $4.4 \pm 0.9$ & $6.0 \pm 0.5$ & $4.8 \pm 0.7^{\star \star \star \star \star *}$ \\
\hline & APRV & $5.6 \pm 1.2$ & $6.4 \pm 1.7$ & $6.4 \pm 1.2$ & $6.4 \pm 1.2$ & $7.6 \pm 1.3^{\star}$ & $7.2 \pm 0.7$ & $8.0 \pm 0.8^{*}$ & $8.2 \pm 0.6^{*}$ \\
\hline & VAFO & $5.4 \pm 0.93$ & $6.2 \pm 0.73$ & $5.8 \pm 0.7$ & $5.6 \pm 0.5$ & $6.8 \pm 0.7$ & $7.2 \pm 0.4$ & $7.0 \pm 0.1$ & $7.2 \pm 0.6$ \\
\hline
\end{tabular}

${ }^{*} p<0.05$ ANOVA RM. Cada grupo versus sus valores basales. ${ }^{* \star} p=0.013$ ANOVA RM, Bonferroni. VAFO versus VCP, APRV. ${ }^{\star \star \star} p<0.05$ ANOVA RM, Bonferroni. VCP versus APRV, VAFO. 
Neumol Cir Torax. 2020; 79 (1): 37-49

Tabla 2: Valores gasométricos en cerdos con inflamación sistémica inducida por LPS tratados con tres modos ventilatorios.

\begin{tabular}{|c|c|c|c|c|c|c|c|c|c|}
\hline Gasometría & Grupo & Basal & $15 \mathrm{~min}$ & 1 hora & 2 horas & 3 horas & 4 horas & 5 horas & 6 horas \\
\hline \multirow{3}{*}{$\begin{array}{l}\mathrm{PaCO}_{2} \\
(\mathrm{mmHg})\end{array}$} & VCP & $43.5 \pm 3.5$ & $38.9 \pm 4.6$ & $45.5 \pm 8.6$ & $39.5 \pm 3.5$ & $43.1 \pm 0.8$ & $38.0 \pm 3.4$ & $38.3 \pm 4.3$ & $36.3 \pm 3.6$ \\
\hline & APRV & $40.9 \pm 4.8$ & $61.9 \pm 18.0^{*}$ & $65.9 \pm 21.8^{*}$ & $44.8 \pm 7.0$ & $43.7 \pm 5.0$ & $48.5 \pm 4.5$ & $35.6 \pm 5.2$ & $37.5 \pm 3.1$ \\
\hline & VAFO & $39.0 \pm 2.7$ & $64.1 \pm 10.5^{\star}$ & $70.5 \pm 11.8^{*}$ & $61.5 \pm 15.2$ & $44.9 \pm 7.9$ & $44.4 \pm 4.7$ & $45.3 \pm 2.0$ & $45.6 \pm 4.1$ \\
\hline \multirow{3}{*}{$\begin{array}{l}\mathrm{PaO}_{2} / \mathrm{FiO}_{2} \\
\text { (ajustado por altitud } \\
2200 \mathrm{MSNM} \text { ) }\end{array}$} & VCP & $328.6 \pm 41.3$ & $442.0 \pm 16.4$ & $421.4 \pm 59.5$ & $342.6 \pm 21.5$ & $336.8 \pm 24.6$ & $298.6 \pm 16.9$ & $345.0 \pm 23.2$ & $291.2 \pm 71.2$ \\
\hline & APRV & $344.7 \pm 30.9$ & $377.6 \pm 38.8$ & $337.2 \pm 46.5$ & $340.6 \pm 44.1$ & $298.2 \pm 45.2$ & $290.2 \pm 58.6$ & $293.2 \pm 42.9$ & $292.2 \pm 54.4$ \\
\hline & VAFO & $360.8 \pm 20.5$ & $284.4 \pm 35.6$ & $322.3 \pm 17.2$ & $400.0 \pm 37.8$ & $376.2 \pm 40.0$ & $361.1 \pm 46.2$ & $360.3 \pm 2.3$ & $372.3 \pm 46.4$ \\
\hline \multirow[t]{3}{*}{ Qt-Qs (\%) } & VCP & $14.6 \pm 3.1$ & $5.8 \pm 1.0$ & $9.6 \pm 2.4$ & $9.2 \pm 1.6$ & $9.4 \pm 1.8$ & $11.8 \pm 1.5$ & $8.8 \pm 2.4$ & $6.6 \pm 1.3$ \\
\hline & APRV & $15.6 \pm 5.5$ & $14.2 \pm 5.6$ & $14.4 \pm 5.4$ & $10.2 \pm 4.0$ & $13.6 \pm 2.4$ & $11.6 \pm 1.5$ & $8.8 \pm 2.4$ & $7.4 \pm 2.5$ \\
\hline & VAFO & $11.2 \pm 0.6$ & $13.8 \pm 0.9^{*}$ & $10.8 \pm 0.7$ & $8.2 \pm 1.6$ & $10.8 \pm 1.4$ & $10.6 \pm 1.4$ & $10.2 \pm 1.6$ & $9.2 \pm 0.7^{\star}$ \\
\hline \multirow{3}{*}{$\begin{array}{l}\text { Glucosa } \\
\mathrm{mg} / \mathrm{dL}\end{array}$} & VCP & $84.0 \pm 7.2$ & $100.2 \pm 11.9$ & $90.2 \pm 12.0$ & $52.4 \pm 13.2$ & $48.6 \pm 17.3$ & $26.8 \pm 11.4^{*}$ & $6.4 \pm 0.5^{\star}$ & $5.4 \pm 1.1^{\star}$ \\
\hline & APRV & $97.8 \pm 13.4$ & $130.0 \pm 45.9$ & $75.8 \pm 13.2^{*}$ & $38.4 \pm 11.4^{*}$ & $21.4 \pm 10.9^{*}$ & $23.6 \pm 16.0^{*}$ & $11.2 \pm 4.2^{*}$ & $12.0 \pm 4.1^{*}$ \\
\hline & VAFO & $88.9 \pm 6.7$ & $89.6 \pm 8.6$ & $77.0 \pm 10.8$ & $51.5 \pm 20.1^{*}$ & $46.8 \pm 11.3^{*}$ & $48.1 \pm 9.6^{*}$ & $61.9 \pm 2.2^{* *}$ & $64.1 \pm 8.0^{\star *}$ \\
\hline \multirow{3}{*}{$\begin{array}{l}\text { Lactato } \\
\mathrm{mmol} / \mathrm{dL}\end{array}$} & VCP & $1.2 \pm 0.6$ & $2.1 \pm 0.7$ & $3.0 \pm 0.8$ & $3.7 \pm 0.8^{*}$ & $4.4 \pm 0.7^{\star}$ & $4.2 \pm 0.7^{\star}$ & $4.8 \pm 0.7^{\star}$ & $5.6 \pm 1.1^{\star}$ \\
\hline & APRV & $0.9 \pm 0.2$ & $1.2 \pm 0.1$ & $2.8 \pm 0.6$ & $4.3 \pm 0.8^{*}$ & $5.0 \pm 0.7^{\star}$ & $6.6 \pm 1.0^{*}$ & $6.9 \pm 0.8^{*}$ & $5.9 \pm 4.1^{*}$ \\
\hline & VAFO & $1.1 \pm 0.3$ & $1.4 \pm 0.2$ & $2.2 \pm 0.3$ & $3.9 \pm 0.9^{*}$ & $4.0 \pm 1.3^{*}$ & $3.1 \pm 1.1$ & $2.1 \pm 0.1^{\star \star \star}$ & $1.7 \pm 0.3^{\star \star *}$ \\
\hline
\end{tabular}

${ }^{*} p<0.05$ ANOVA RM. Cada grupo versus sus valores basales. ${ }^{* *} p<0.05=$ ANOVA RM, Bonferroni. VCP versus APRV, VAFO. ${ }^{* \star} p<0.05$ ANOVA RM, Bonferroni. VAFO versus VCP, APRV. Promedio $\pm \mathrm{DE}=$ Desviación estándar.

vó durante todo el estudio en los grupos I y II $(\mathrm{p}=0.01$, ANDEVA-MR, Bonferroni) (Tabla 1).

Las RVS en todos los grupos de estudio disminuyeron durante las dos primeras horas del estudio y a partir de la tercera hora retornaron a valores similares a los basales. Al comparar los valores observados dentro de cada grupo y entre grupos no mostraron cambios significativos ( $p>0.05$ ANDEVA-MR).

Todos los animales mostraron hipertensión pulmonar ( $p$ $=0.02$ ANDEVA-MR, Bonferroni) (Tabla 1).

Las RVP se incrementaron en comparación con sus valores basales $(p<0.05$ ANDEVA-MR) a partir de los 15 minutos posinicio de los diferentes modos ventilatorios y se mantuvo así hasta el final del estudio. Al comparar entre grupos no se observaron diferencias significativas $(p=0.75$ ANDEVA-MR) (Tabla 1).

La PVC en todos los grupos se mantuvo dentro de los parámetros de referencia y se preservó así hasta concluir el estudio en los grupos II y III; no obstante, en el grupo I, la PVC disminuyó a partir de las tres horas del estudio, pero esta disminución sólo fue significativa a las seis horas al comparar con sus valores basales y grupos II y III ( $p=$ 0.01 ANDEVA-MR, Bonferroni).

\section{Hallazgos gasométricos}

La $\mathrm{PaCO}_{2}$ en el grupo I se mantuvo dentro de los parámetros de referencia. En los grupos II y III se incrementó a los
15 minutos posteriores al inicio de los diferentes modos ventilatorios al compararlas con sus valores basales $(p<$ 0.05, ANDEVA-MR); sin embargo, regresó a sus valores de referencia a las tres horas en ambos grupos de estudio (Tabla 2).

$\mathrm{La} \mathrm{PaO}_{2} / \mathrm{FiO}_{2}$ en los tres grupos se conservó dentro de los parámetros de referencia durante todo el estudio y no se observaron diferencias entre grupos ( $p>0.05$, ANDEVAMR) (Tabla 2).

La glucosa en la gasometría arterial disminuyó en todos los grupos de estudio a las dos horas de haber iniciado los diferentes modos ventilatorios ( $p<0.05$, ANDEVA-MR) versus sus valores basales ( $p<0.05$, ANDEVA-MR) y así se mantuvo en los grupos I y II hasta concluir el estudio. Mientras tanto, en el grupo III se elevó a las cinco y seis horas de la AMV y aunque este incremento no alcanzó a sus valores normales, éstos fueron superiores a los de los grupos I y II ( $p=0.02$, ANDEVA-MR, Bonferroni) (Tabla 2).

El lactato en los tres grupos de estudio se incrementó a las dos horas posteriores al inicio de cada modo ventilatorio ( $p<0.05$, ANDEVA-MR) en comparación con sus valores basales y se preservó hasta concluir el estudio en los grupos I y II; pero en el grupo III éste disminuyó a las cuatro horas, y a las cinco horas regresó a los valores de referencia a diferencia de los otros dos grupos $(p=0.01$, ANDEVA-MR, Bonferroni) (Tabla 2). 


\section{Hallazgos de la mecánica pulmonar}

En ambos grupos la $\mathrm{P}_{\text {pico }}$ se incrementó significativamente a las tres horas de iniciar la terapia con los diferentes modos ventilatorios versus sus valores basales $(p>0.05$, ANDEVA-MR) y se mantuvo así durante todo el tiempo de la investigación. Al comparar entre grupos no se observaron diferencias ( $p=0.783$, ANDEVA-MR, Bonferroni) (Tabla 3).

La Paw en los grupos II y III se incrementó a los 15 minutos después de haber iniciado cada modo ventilatorio en comparación con sus valores de referencia y así se conservó durante todo el estudio ( $p<0.05$, ANDEVA-MR). El grupo I no mostró cambios. Al comparar entre grupos los valores del grupo I fueron menores que los de los otros, desde los 15 minutos hasta el final del estudio $(p=0.01$, ANDEVAMR, Bonferroni) (Tabla 3).

Al valorar la $C_{d y n}$ se observó que en el grupo I, disminuyó significativamente a las tres horas de haber iniciado cada modo ventilatorio versus valores basales $(p<0.05$ ANDEVA-MR) y se preservó hasta el final del estudio. Al comparar entre grupos los valores en el grupo I fueron menores que los del grupo II ( $p=0.024$ ANDEVA-MR, Bonferroni) (Tabla 3).

El $\Delta \mathrm{P}$ en ambos grupos aumentó al compararlos con sus valores basales. En el grupo II se presentó a los 15 minutos ( $p<0.05$, ANDEVA-MR) a partir del cambio del modo ventilatorio; mientras que en el grupo I fue a la primera hora y así se mantuvieron hasta el final del estudio ( $p<0.05$, ANDEVA-MR). Al comparar entre grupos no se observaron diferencias ( $p=0.55$, ANDEVAMR) (Tabla 3).

El trabajo respiratorio (WOBv) sólo se acrecentó en el grupo I entre las tres y cinco horas ( $p<0.05$, ANDEVA-MR) del estudio; no obstante, al comparar entre grupos este incremento no fue significativo ( $p=0.55$, ANDEVA-MR, Bonferroni) (Tabla 3).

\section{Hallazgos radiológicos}

Ninguno de los animales del estudio mostró cambios radiológicos durante todo el estudio (Figura 1).

\section{Hallazgos microscópicos}

Se muestra el corte histológico de pulmón sano como referencia en la Figura 2A. Histológicamente, los animales del grupo I mostraron hemorragia y congestión leve (Figura 2B). En el grupo II la hemorragia y congestión fue moderada (Figura 2C); mientras que en el grupo III estos hallazgos fueron ausentes ( $p<0.05$, Kruskal-Wallis) (Figura 2D). También en los tres grupos se observó colapso e inflamación con predominio de polimorfos nucleares (PMN) y lesiones leves a moderadas ( $p>0.05$, Kruskal-Wallis). En ningún grupo se presentó edema pulmonar.

Tabla 3: Valores de la mecánica pulmonar en cerdos con inflamación sistémica inducida por LPS tratados con tres modos ventilatorios.

\begin{tabular}{|c|c|c|c|c|c|c|c|c|c|}
\hline $\begin{array}{l}\text { Mecánica } \\
\text { pulmonar }\end{array}$ & Grupo & Basal & $15 \mathrm{~min}$ & 1 hora & 2 horas & 3 horas & 4 horas & 5 horas & 6 horas \\
\hline \multirow{3}{*}{$\begin{array}{l}\mathrm{P}_{\text {pico }} \\
\left(\mathrm{cmH}_{2} \mathrm{O}\right)\end{array}$} & VCP & $17.4 \pm 0.8$ & $16.2 \pm 1.0$ & $18.8 \pm 2.3$ & $20.2 \pm 2.6$ & $22.6 \pm 2.1$ & $24.4 \pm 2.1$ & $24.6 \pm 2.0^{*}$ & $24.8 \pm 1.7^{*}$ \\
\hline & APRV & $16.2 \pm 3.5$ & $21.4 \pm 1.8$ & $21.2 \pm 3.7$ & $18.0 \pm 2.9$ & $22 \pm 1.1$ & $24.0 \pm 1.5$ & $24.4 \pm 1.1$ & $24.2 \pm 1.2$ \\
\hline & VAFO & & $\mathrm{N} / \mathrm{A}$ & $N / A$ & $\mathrm{~N} / \mathrm{A}$ & $N / A$ & $N / A$ & $\mathrm{~N} / \mathrm{A}$ & $\mathrm{N} / \mathrm{A}$ \\
\hline \multirow{3}{*}{$\begin{array}{l}\text { Paw } \\
\left(\mathrm{cmH}_{2} \mathrm{O}\right)\end{array}$} & VCP & $9.2 \pm 0.2$ & $8.8 \pm 0.5^{\star *}$ & $8.8 \pm 0.6^{* *}$ & $9.0 \pm 0.6^{\star *}$ & $9.8 \pm 0.6^{\star *}$ & $10.4 \pm 0.7^{\star \star}$ & $10.4 \pm 0.6^{\star \star}$ & $10.8 \pm 0.8^{\star \star}$ \\
\hline & APRV & $6.8 \pm 1.5$ & $14.0 \pm 2.0^{*}$ & $15.8 \pm 2.5^{\star}$ & $15.8 \pm 2.2^{*}$ & $17.0 \pm 1.3^{\star}$ & $17.6 \pm 1.1^{*}$ & $17.6 \pm 0.5^{\star}$ & $18.2 \pm 1.1^{*}$ \\
\hline & VAFO & $8.6 \pm 0.4$ & $13.2 \pm 0.7^{\star}$ & $14.0 \pm 0.1^{*}$ & $14.0 \pm 0.1^{*}$ & $13.9 \pm 0.2^{*}$ & $14.1 \pm 0.1^{*}$ & $14.1 \pm 0.1^{*}$ & $14.1 \pm 0.1^{*}$ \\
\hline \multirow{3}{*}{$\begin{array}{l}\mathrm{C}_{\text {din }}(\mathrm{mL} / \\
\left.\mathrm{CmH}_{2} \mathrm{O}\right)\end{array}$} & VCP & $16.2 \pm 2.3$ & $15.0 \pm 3.5$ & $15.2 \pm 6.0$ & $9.0 \pm 5.2$ & $8.8 \pm 2.2^{*}$ & $7.2 \pm 4.3^{*}$ & $7.8 \pm 4.5^{\star}$ & $7.2 \pm 2.3^{*}$ \\
\hline & APRV & $18.0 \pm 3.9$ & $26.8 \pm 12.2$ & $18.2 \pm 2.2$ & $16.4 \pm 4.4$ & $15.8 \pm 1.2$ & $15.0 \pm 1.1^{\star \star \star}$ & $15.0 \pm 1.5^{\star \star \star}$ & $14.4 \pm 1.0^{\star \star \star}$ \\
\hline & VAFO & & $\mathrm{N} / \mathrm{A}$ & $N / A$ & $\mathrm{~N} / \mathrm{A}$ & $\mathrm{N} / \mathrm{A}$ & $N / A$ & $N / A$ & $\mathrm{~N} / \mathrm{A}$ \\
\hline \multirow[t]{3}{*}{$\Delta \mathrm{P}\left(\mathrm{cmH}_{2} \mathrm{O}\right)$} & VCP & $12.6 \pm 2.1$ & $11.4 \pm 1.9$ & $14.0 \pm 4.7$ & $15.4 \pm 5.5$ & $17.8 \pm 4.4$ & $19.6 \pm 4.4$ & $19.8 \pm 4.2$ & $20.8 \pm 2.4^{*}$ \\
\hline & APRV & $13.2 \pm 6.4$ & $20.0 \pm 3.4^{*}$ & $20.2 \pm 8.9$ & $16.6 \pm 7.6$ & $18.0 \pm 4.5$ & $18.2 \pm 5.2$ & $19.0 \pm 6.0$ & $18.8 \pm 2.4$ \\
\hline & VAFO & & $N / A$ & $N / A$ & $N / A$ & $\mathrm{~N} / \mathrm{A}$ & $N / A$ & $N / A$ & $\mathrm{~N} / \mathrm{A}$ \\
\hline \multirow[t]{3}{*}{ WOBv (J/L) } & VCP & $0.7 \pm 0.2$ & $1.0 \pm 0.1$ & $1.0 \pm 0.1$ & $1.1 \pm 0.1$ & $1.2 \pm 0.1^{*}$ & $1.4 \pm 0.1^{*}$ & $1.4 \pm 0.1^{*}$ & $1.4 \pm 0.1$ \\
\hline & APRV & $1.9 \pm 1.8$ & $2.6 \pm 1.6$ & $1.0 \pm 0.3$ & $0.7 \pm 0.3$ & $0.5 \pm 1.2$ & $2.1 \pm 1.7$ & $2.1 \pm 1.7$ & $3.6 \pm 2.2$ \\
\hline & VAFO & & $\mathrm{N} / \mathrm{A}$ & $\mathrm{N} / \mathrm{A}$ & $\mathrm{N} / \mathrm{A}$ & $\mathrm{N} / \mathrm{A}$ & $\mathrm{N} / \mathrm{A}$ & $\mathrm{N} / \mathrm{A}$ & $\mathrm{N} / \mathrm{A}$ \\
\hline
\end{tabular}

${ }^{\star} p<0.05$ ANOVA RM. Cada grupo versus sus valores basales. ${ }^{* \star} p<0.05$ ANOVA RM, Bonferroni. VCP versus APRV, VAFO. ${ }^{\star \star \star} p<0.05$ ANOVA RM, Bonferroni. VAFO APRV versus VCP, APRV. 

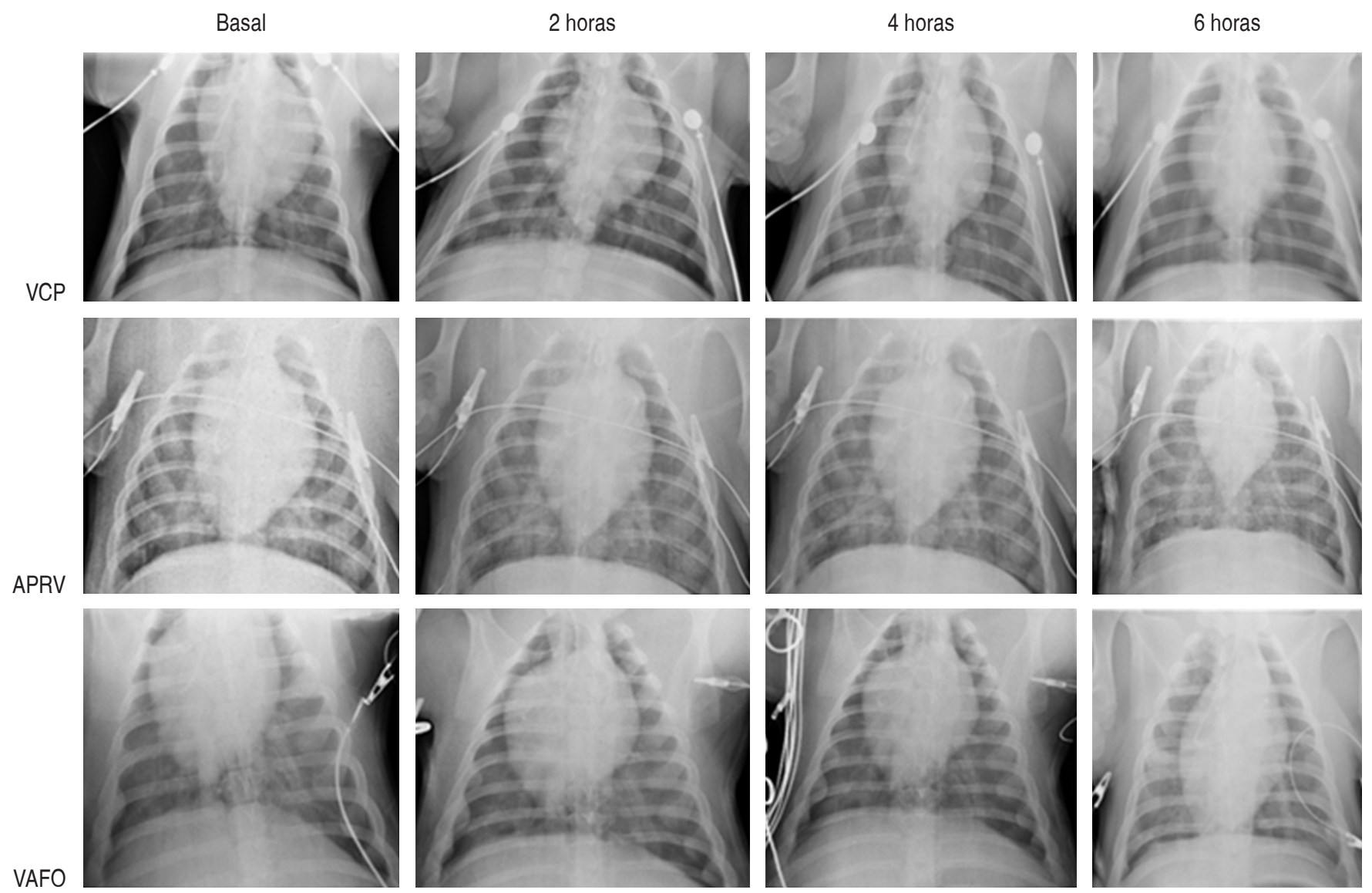

Figura 1: Radiografías de tórax ventrodorsal, durante la ventilación mecánica en un animal representativo de cada uno de los tres grupos.

\section{DISCUSIÓN}

La sepsis es una infección generalizada caracterizada por una inflamación sistémica en la cual los pacientes que la padecen llegan a presentar LPA leve y aunque no cumplan con los criterios de SIRA requieren de $\mathrm{AMV}, 2,6,7$ con estrategias de protección pulmonar para evitar la progresión del mismo; pero si no se lleva a cabo de forma apropiada puede ocasionar $\mathrm{VILI}{ }^{10}$

Hasta la fecha no existe un modo ventilatorio en específico para ser utilizado en la terapia de la sepsis, ${ }^{10}$ ya que se ha demostrado que los modos ventilatorios más utilizados (VCV y VCP) ${ }^{11}$ pueden dañar la arquitectura pulmonar, la función y la hemodinamia; ${ }^{12,13}$ por lo que es necesario buscar y adecuar nuevos modos ventilatorios que eviten esto y no incrementen las lesiones pulmonares ocasionadas por la inflamación sistémica inducida por la sepsis. Con base en lo anterior, el objetivo de este estudio fue comparar el efecto de la AMV en modo VCP, APRV y VAFO como única terapia sobre la hemodinamia, gasometría, mecánica e histología pulmonar en un modelo porcino de inflamación sistémica inducida por LPS E. coli.
En este estudio utilizamos el modelo de inflamación sistémica producida por LPS E. coli, debido a que la administración vía sistémica del mismo ocasiona una reacción inflamatoria rápida, ${ }^{25}$ mediada principalmente por los receptores tipo Toll 4 (TLR-4), que al ponerse en contacto con el LPS ocasionan una respuesta inflamatoria rápida con alteraciones fisiológicas ${ }^{25-27}$ como taquicardia, hipotensión arterial sistémica, hipertensión pulmonar y disminución del GC como se observó en este estudio. También utilizamos este modelo porque la infusión del LPS a través de un catéter venoso central tiene la ventaja de que los pulmones sean el primer endotelio afectado y ocasione cambios a nivel pulmonar; ${ }^{28,29}$ sin embargo, su uso no siempre ocasiona SIRA como se observó en este estudio y concuerda con lo descrito por Müller-Leisse y su grupo. ${ }^{29}$ Además, la ausencia de SIRA pudo ser ocasionada porque al inicio del estudio se utilizó una $\mathrm{FiO}_{2}$ de 100\% para asegurar altas concentraciones de $\mathrm{PaO}_{2}$ previas al daño por LPS, lo cual pudo atenuar la inducción del mismo, como se ha descrito en otros estudios en los que la administración de $\mathrm{FiO}_{2}$ al $100 \%$ en un período de seis horas favorece la menor presencia de SIRA. ${ }^{30-33}$ 
Neumol Cir Torax. 2020; 79 (1): 37-49

En este estudio, los hallazgos hemodinámicos, hiperlactatemia, hipoglucemia y cambios en la $\mathrm{PaCO}_{2}$ observados a la primera hora posadministración del LPS E. coli (o inicio de los diferentes modos ventilatorios) demostraron que todos los animales desarrollaron inflamación sistémica, como lo han descrito otros autores que han hecho estudios en modelos porcinos, caninos, primates no humanos y ratas, y han observado estos cambios entre las 1-1.5 horas después de la administración del LPS. ${ }^{1,2,34,35}$ Empero, nuestros hallazgos no concuerdan con lo reportado por Herrera y colaboradores $^{23}$ quienes observaron estos cambios entre los 2-10 minutos posadministración del LPS en un modelo experimental canino.

En este estudio el mejor comportamiento del GC y PAM en el grupo III probablemente fue ocasionado porque la Paw utilizada en este modo de AMV incrementó la presión intratorácica y la acumulación sanguínea en el lecho vascular pulmonar, el cual actuó como un reservorio para el ventrículo izquierdo, lo que aumentó la precarga y disminuyó la poscarga del mismo a diferencia de los otros dos modos ventilatorios en los que hay aumento en la poscarga de éste y concuerda con lo observado por Osiovich, ${ }^{18}$ Karmrodt, ${ }^{19}$ Traverse, ${ }^{36}$ David, ${ }^{37}$ Smailys, ${ }^{38}$ y sus respectivos grupos de investigación, quienes reportaron este efecto de la AMV en modo VAFO al utilizar Paw mayores a $18 \mathrm{cmH}_{2} \mathrm{O}$ y sus efectos sobre el pulmón y corazón en comparación con otros modos ventilatorios. Estos hallazgos también coinciden con lo reportado por
Gu y colaboradores ${ }^{39}$ quienes realizaron un metaanálisis en el que compararon la VAFO contra la ventilación protectora en pacientes con SIRA, y observaron que este modo de AMV no incrementa significativamente el riesgo de hipotensión en comparación con el modo convencional. Por otro lado, los cambios mostrados por el grupo I se originaron por el efecto de la PEEP y la Paw sobre la presión transpulmonar; mientras que en el grupo II se produjeron porque el mayor tiempo inspiratorio incrementó la presión transpulmonar y la poscarga del ventrículo izquierdo (Emr y su grupo ${ }^{33}$ y Zhou y colegas). ${ }^{40}$ Nuestros hallazgos también concuerdan con lo observado por Derdak y colaboradores ${ }^{41}$ quienes evaluaron la efectividad y seguridad del VAFO contra la ventilación convencional en pacientes con SIRA y observaron que la FC, GC y PAM permanecieron normales a las 72 horas de ser sometidos a cada modo ventilatorio. Además, concuerdan con lo descrito por Roosens ${ }^{42}$ Osiovich, ${ }^{18}$ y Smailys ${ }^{38}$ y sus respectivos equipos de trabajo, quienes valoraron el efecto del modo ventilatorio VCP en modelos experimentales sanos y con SIRA. También cabe mencionar que en este estudio, posiblemente en los grupos I y II, la PAM no retomó sus valores basales porque sólo se aplicó como tratamiento la AMV y no se administró ninguna terapia de líquidos, ni vasopresores, por lo que continuaron las anormalidades en la perfusión. ${ }^{43}$

Con relación a las RVS, después de someter a los cerdos a los diferentes modos ventilatorios, disminuyeron

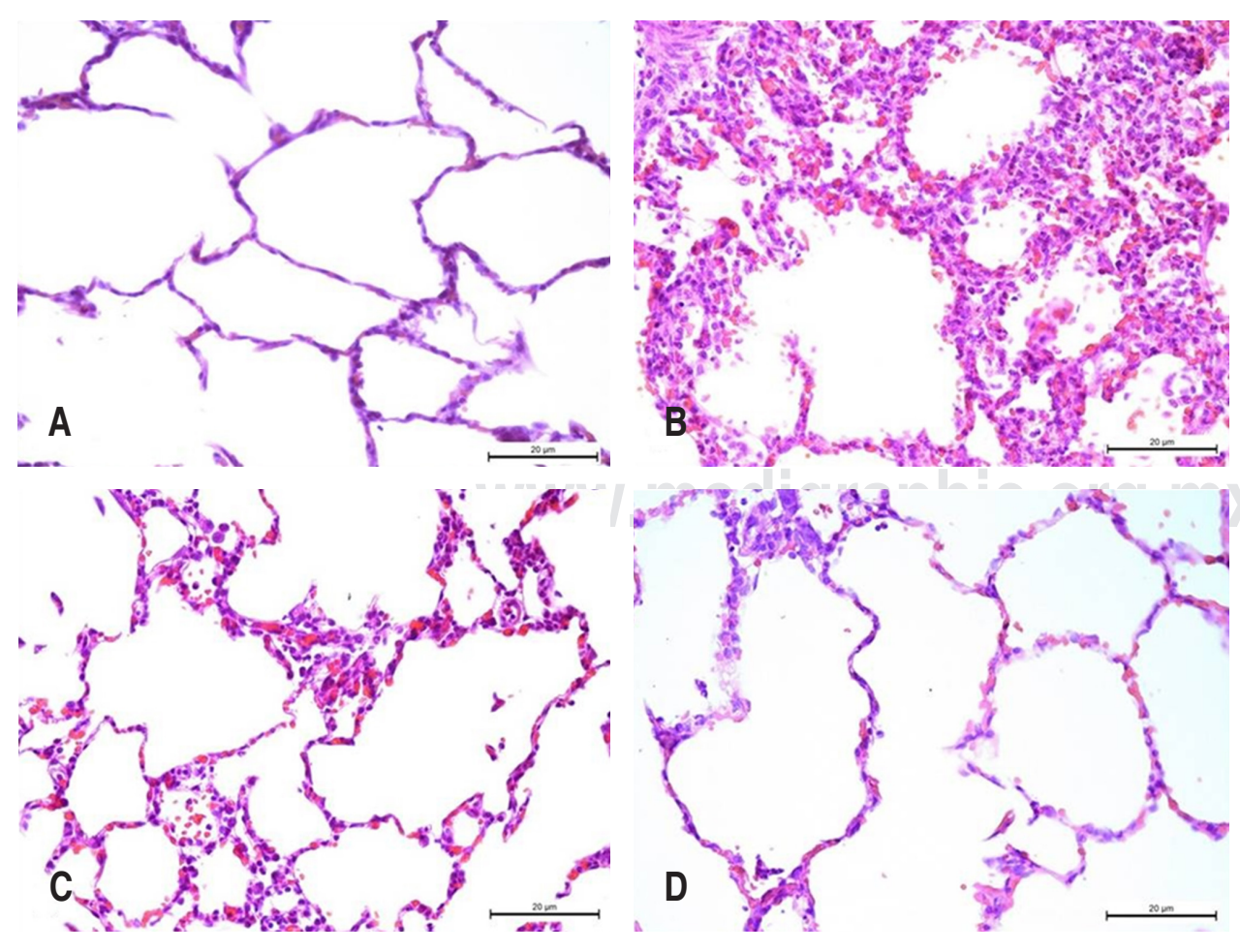

Figura 2:

Fotomicrografías en las que se observa: A) pulmón sano, B) inflamación, hemorragia, congestión y colapso severo en un animal del grupo I; C) moderada inflamación, hemorragia, congestión y colapso en un cerdo del grupo II y D) mismos cambios, pero de forma leve, en el grupo III (HE 10x). 
durante las primeras horas porque el LPS E. coli al producir la respuesta inflamatoria ocasiona vasodilatación y disminución de las RVS. ${ }^{25-27}$ Sin embargo, retornaron sus valores basales después de aplicar los diferentes modos ventilatorios por los efectos provocados sobre la presión intratorácica ocasionados por cada modo ventilatorio, como describieron anteriormente Osiovich, ${ }^{18}$ Karmrodt, $^{19}$ Traverse, ${ }^{36}$ David $^{37}$ y Smailys ${ }^{38}$ y sus respectivos grupos de investigación.

Los incrementos en las PMAP y RVP observados en los modos ventilatorios de APRV y VAFO probablemente fueron originados porque además del daño provocado por el LPS E. coli, estos modos ventilatorios ocasionaron incrementos en la presión transpulmonar y, como consecuencia de estos cambios, en la precarga y poscarga cardíaca, lo cual concuerda con lo descrito por otros autores que han estudiado el uso de la AMV en modo APRV y VAFO en pacientes con SIRA ${ }^{14,33}$ y en modelos animales de VILI $^{40} y$ sepsis. ${ }^{18}$ Asimismo, los cambios observados en el grupo I fueron ocasionados por la PEEP. ${ }^{38}$

La POAP se mantuvo dentro de valores normales porque en este tiempo del estudio la precarga del ventrículo izquierdo se encontraba normal, como lo observado por otros autore $\mathrm{s}^{44}$ que estudiaron la utilidad de los modos ventilatorios VCP y APRV en perros con LPA y mencionan que la POAP no cambia durante éste. Nuestros hallazgos también concuerdan con lo reportado por Putensen y colegas $^{45}$ que estudiaron los efectos de la AMV en modo VCP versus APRV en pacientes con trauma craneoencefálico sin SIRA y observaron que la POAP no se altera debido a que la actividad respiratoria espontánea no tiene efectos hemodinámicos, pues no disminuye la presión intratorácica. En contraste, los resultados de este estudio no concuerdan con lo observado por Fort y su equipo, ${ }^{46}$ quienes indican que en pacientes con SIRA observaron que la POAP se incrementa de forma leve pero significativa a las tres horas de haber sometido a los pacientes a VAFO.

Con respecto a la PVC, en nuestro estudio a pesar de que no se administraron líquidos y se aplicó AMV, ésta se mantuvo dentro de los parámetros normales como se recomienda durante la sepsis (mantener una PVC entre 8-12 mmHg sin AMV y 12-15 mmHg con AMV), ${ }^{47}$ lo que podría sugerir que en el modo ventilatorio ninguno de los tres modos ventilatorios afecta el mantenimiento de la misma.

Durante todo el estudio, los Qt-Qs, $\mathrm{PaCO}_{2}$ y $\mathrm{PaO}_{2} /$ $\mathrm{FiO}_{2}$ se mantuvieron dentro de los parámetros normales en todos los grupos, debido a que la ventilación mecánica redujo el colapso alveolar e incrementó la capacidad residual funcional, lo cual mejoró el equilibrio entre ventilación y perfusión..$^{48}$ Nuestros hallazgos concuerdan con lo observado en varios estudios clínicos y experimentales de sepsis y SIRA en los que se menciona que la mayor Paw utilizada en APRV y VAFO en comparación con los modos convencionales, así como el uso de $\mathrm{FiO}_{2}$ superiores a $60 \%$ mejoran la $\mathrm{PaO}_{2} / \mathrm{FiO}_{2}$ antes de las 24 horas de haberse iniciado el modo ventilatorio. ${ }^{18-20,41,45,48}$

Por otro lado, la hiperglucemia observada en todos los grupos al inicio del estudio fue originada porque el LPS ocasionó un estado de estrés como el que sufren los pacientes con sepsis y choque séptico que con frecuencia cursan con un estado hipermetabólico caracterizado por elevación del gasto energético, resistencia celular a la acción de la insulina y elevación de glucemia. ${ }^{49}$ Asimismo, la disminución gradual de la misma en el grupo II, a partir de la primera hora de haber iniciado la transición del modo ventilatorio, quizás se debió a que el menor uso de fármacos vasopresores y anestésicos produce un estímulo adrenérgico que libera catecolaminas e inducen desbalances en los niveles de glucosa. ${ }^{50,51}$ Mientras que la disminución de la glucosa, a las dos horas de haber realizado la transición del modo ventilatorio en el grupo I, quizá se originó porque la lesión ocasionada por el LPS fue evolucionando y como no hubo terapia de líquidos no se administró glucosa adicional. Además, el incremento de ésta en el grupo III (aunque no alcanzó sus valores basales) durante las dos últimas horas del estudio fue, probablemente, porque el modo ventilatorio incrementó la perfusión tisular y disminuyó el consumo de glucosa. A la fecha no hay estudio que respalden estos hallazgos.

La hiperlactatemia producida se debe a que en el choque séptico la hipotensión sistémica provoca hipoxia celular, metabolismo anaerobio, déficit de piruvato deshidrogenasa y la disminución en la depuración del lactato en el hígado. Aunque, la menor hiperlactatemia mostrada por los animales que recibieron terapia con el modo VAFO, posiblemente fue debido a que éstos presentaron mejor perfusión tisular y depuración del lactato en el hígado como lo describió Nguyen y colaboradores al estudiar los efectos de la disminución temprana del lactato en pacientes con choque séptico severo. ${ }^{52}$

Por las características de los ventiladores utilizados, la mecánica pulmonar sólo puede ser comparada entre los grupos I y II, con excepción de la Paw que sí se obtuvo en todos los grupos.

El incremento en la $\mathrm{P}_{\text {pico }}$ en ambos grupos de estudio fue originado porque durante la sepsis el SIRA presenta colapso alveolar y pérdida de distensibilidad pulmonar. En el grupo I estos cambios se originaron ya que el PEEP utilizado en este estudio sólo fue de $5 \mathrm{cmH}_{2} \mathrm{O}$, el cual no fue suficiente para evitar estas alteraciones como lo observaron Kaisers y colegas ${ }^{53}$ y Ziebart y su equipo ${ }^{2}$ en un modelo experimental de sepsis en el que se aplicó PEEP de 5 y $10 \mathrm{cmH}_{2} \mathrm{O}$. Mientras que en grupo II se observó el mismo efecto al aplicar una $\mathrm{P}_{\text {baja }}$ de $5 \mathrm{CmH}_{2} \mathrm{O}$. 
Neumol Cir Torax. 2020; 79 (1): 37-49

Con respecto al mayor incremento observado en la Paw en los grupos tratados con APRV y VAFO (que es la única que se puede comparar) inmediatamente después de haber realizado la transición del modo ventilatorio y durante todo el estudio coincide con lo descrito por Maxwell y colaboradores ${ }^{54}$ y Siau y su grupo de investigación, ${ }^{55}$ quienes mencionan que estos modos ventilatorios se caracterizan por aumentar la Paw con el fin de favorecer el reclutamiento alveolar, tal como lo observaron en pacientes traumatizados que desarrollaron SIRA ventilados con estos modos ventilatorios.

En este estudio en el grupo I, la $C_{d y n}$ disminuyó posiblemente por la presencia de edema y colapso alveolar (que se observó histológicamente) que evitaron la libre expansión del pulmón y de la cavidad torácica. No obstante, ésta no pudo recuperarse postransición del modo ventilatorio debido a que se incrementó la $\mathrm{P}_{\text {pico' }}$ además de que el PEEP utilizado en este estudio no fue suficiente para mantenerla dentro de los límites normales. Nuestros hallazgos no coinciden con lo descrito por otros autores que utilizaron PEEP más elevado y no observaron estos cambios. ${ }^{45,56}$ Por otro lado, en el grupo tratado con APRV, la $C_{d y n}$ se mantuvo durante todo el estudio porque la presencia de colapso pulmonar fue menor; además, porque este modo ventilatorio permite respiraciones espontáneas que favorecen el reclutamiento alveolar por el incremento que se produce de presiones y tiempos durante la relación I:E, lo cual concuerda con lo observado por Putensen y colaboradores ${ }^{45}$ y Lara-Cruz y colegas ${ }^{56}$ al utilizar APRV en pacientes politraumatizados y sépticos, respectivamente.

El mantenimiento del $\triangle \mathrm{P}$ y del WOBv en ambos grupos de estudio fue originado porque durante la AMV hubo un equilibrio entre la $P_{\text {pico }}$ y el PEEP en el modo de VCP o $P_{\text {baja }}$ en APRV, lo cual evitó una sobredistensión y daño pulmonar originado por la $\mathrm{AMV}, 57,58$ así como una adecuada $\mathrm{PaO}_{2} /$ $\mathrm{FiO}_{2}$. Sin embargo, no podemos comparar los hallazgos de esta investigación con otras, debido a que no existen estudios que describan el trabajo respiratorio en sepsis utilizando estos modos ventilatorios.

No se observaron cambios radiológicos, ya que el tiempo de estudio fue corto y no permitió ver la presencia de lesiones compatibles con SIRA, tampoco se observó ninguna alteración radiológica compatible con el uso de la AMV.

Histológicamente, en todos los grupos la inflamación con polimorfonucleares, hemorragia, congestión y edema fue originado porque el LPS provoca acumulación de neutrófilos y daño tisular. ${ }^{1,2,25-27}$ No obstante, los animales del grupo II presentaron mayor inflamación debido a que desarrollaron mayor congestión vascular y hemorragia en el espacio alveolar, lo cual ocasionó mayor salida de célu- las inflamatorias al mismo. Estos hallazgos no concuerdan con lo descrito por otros autore ${ }^{33}$ que compararon el uso del VCV (con volumen tidal de $10 \mathrm{~mL} / \mathrm{kg}$ ) versus APRV en pulmones de rata sanas para evaluar cuál de estos modos ventilatorios produce menor daño y observaron que el APRV disminuye la presencia de estas lesiones. Por otra parte, nuestros resultados se relacionan con lo observado por Roy y otros autore ${ }^{13}$ quienes compararon APRV contra VCV con bajos volúmenes tidales $(6 \mathrm{~mL} / \mathrm{kg})$ en cerdos con sepsis peritoneal por punción cecal y describieron que el modo APRV conserva la arquitectura alveolar y evita el colapso, pero no evita la dilatación linfática. Por otro lado, en los animales tratados con VCP se presentó mayor grado de colapso, ya que en este modo ventilatorio el PEEP utilizado no fue suficiente para mantener abiertos los alvéolos como lo describió Albert y colaboradores ${ }^{59}$ al estudiar el papel de la ventilación mecánica y la disfunción del surfactante como causa de ARDS.

La menor inflamación, hemorragia, congestión y colapso histológico observada en los cerdos tratados con VAFO probablemente fueron ocasionados por el efecto del modo ventilatorio que mantiene una Paw alta debido a la oscilación prolongada, lo que disminuye el daño mecánico y favorece el reclutamiento alveolar que previene y evita el colapso. ${ }^{60}$ Estos hallazgos concuerdan con lo reportado por $\mathrm{Fu}$ y su equipo ${ }^{12}$ quienes observaron esto a las 24 y 48 horas al comparar el modo ventilatorio VAFO y VCP en cerdos con lesión pulmonar aguda ocasionada por solución salina caliente. Asimismo, concuerdan con lo observado por Siau y colaboradore $\mathrm{s}^{55}$ quienes al realizar una revisión de diferentes estudios de la utilidad de la VAFO y APRV en LPA, así como en SIRA experimental y clínica, mencionan que estos cambios histológicos pueden deberse a los gradientes de presión de cada alvéolo. Otros autores ${ }^{60}$ mencionan que en el modo VAFO se producen dos fenómenos denominados dispersión de Taylor y efecto pendelluft que generan perfiles de velocidad asimétrica, los cuales producen un flujo constante y retención de aire, lo que ocasiona menor congestión y hemorragia, además de reclutamiento alveolar que previene y evita el colapso.

\section{CONCLUSIONES}

Los modos ventilatorios VCP, APRV y VAFO al ser utilizados como único soporte en las fases iniciales de sepsis inducida con LPS E. coli en cerdos mantienen los valores gasométricos y ventilatorios dentro de los parámetros normales. Sin embargo, el modo VAFO favorece la recuperación de los valores hemodinámicos sistémicos y produce menor daño histológico. Los modos VCP y APRV presentaron mayor grado de hipotensión sistémica y alteraciones histológicas. 


\section{Agradecimientos}

A. Guzmán, agradece a CONACyT por su beca durante sus estudios de doctorado en la Universidad Nacional Autónoma de México. Agradecemos a Antonia Luna Flores, Roberto Rueda Juárez, Marcelino Alonso Gómez y Sergio García Martínez por su apoyo durante esta investigación.

\section{REFERENCIAS}

1. Zhang Y, Gao J, Wang CJ, Zhou LJ, Fang XZ, Yang LQ. Low tidal volume ventilation preconditioning ameliorates lipopolysaccharideinduced acute lung injury in rats. Acta Anaesthesiol Scand 2016;60(6):780-789. doi: 10.1111/aas.12691

2. Ziebart A, Hartmann EK, Thomas R, et al. Low tidal volume pressure support versus controlled ventilation in early experimental sepsis in pigs. Respir Res 2014;15:101. https://doi.org/10.1186/s12931-0140101-6

3. Wheeler AP, Bernard GR. Acute lung injury and the acute respiratory distress syndrome: a clinical review. Lancet 2007;369(9572):15531564. https://doi.org/10.1016/S0140-6736(07)60604-7

4. Shankar-Hari M, Phillips GS, Levy ML, et al.; Sepsis Definitions Task Force. Sepsis definitions task force. Developing a new definition and assessing new clinical criteria for septic shock: for the third international consensus definitions for sepsis and septic shock (Sepsis-3). JAMA 2016;315(8):775-787. https://doi.org/10.1001/ jama.2016.0289

5. Seymour CW, Liu VX, Iwashyna TJ, et al. Assessment of clinical criteria for sepsis: for the Third International Consensus Definitions for Sepsis and Septic Shock (Sepsis-3). JAMA 2016;315(8):762-774. https://doi.org/10.1001/jama.2016.0288

6. Fan E, Stewart TE. New modalities of mechanical ventilation: high-frequency oscillatory ventilation and airway pressure release ventilation. Clin Chest Med 2006;27(4):615-625. Abstract viii-ix. https:// doi.org/10.1016/j.ccm.2006.06.008

7. Sevransky JE, Levy MM, Marini JJ. Mechanical ventilation in sepsisinduced acute lung injury/acute respiratory distress syndrome: an evidence-based review. Crit Care Med 2004;32(11 Suppl):S548-S553. https://doi.org/10.1097/01.ccm.0000145947.19077.25

8. Meredith W, Rutledge R, Fakhry SM, Emery S, Kromhout-Schiro S. The conundrum of the Glasgow Coma Scale in intubated patients: a linear regression prediction of the Glasgow verbal score from the Glasgow eye and motor scores. J Trauma 1998;44(5):839-844. https:// doi.org/10.1097/00005373-199805000-00016

9. Carrasco Loza R, Villamizar Rodríguez G, Medel Fernández N. Ventilator-Induced Lung Injury (VILI) in Acute Respiratory Distress Syndrome (ARDS): volutrauma and molecular effects. Open Respir Med J 2015;9:112-119. https://doi.org/10.2174/1874306401509010112

10. Dellinger RP, Levy MM, Rhodes A, et al:; Surviving Sepsis Campaign Guidelines Committee Including the Pediatric Subgroup. Surviving sepsis campaign: international guidelines for management of severe sepsis and septic shock, 2012. Intensive Care Med 2013;39(2):165228. https://doi.org/10.1007/s00134-012-2769-8

11. Kredel M, Bierbaum D, Lotz C, Küstermann J, Roewer N, Muellenbach RM. Therapy of acute respiratory distress syndrome; survey of German ARDS centers and scientific evidence. Anaesthesist 2015;64(4):277-285. https://doi.org/10.1007/s00101015-0010-1
12. Fu W, Qin X, You C, Meng Q, Zhao Y, Zhang Y. High frequency oscillatory ventilation versus conventional ventilation in a newborn piglet model with acute lung injury. Respir Care 2013;58(5):824-830. https://doi.org/10.4187/respcare.01972

13. Roy S, Habashi N, Sadowitz B, et al. Early airway pressure release ventilation prevents ARDS-a novel preventive approach to lung injury. Shock 2013;39(1):28-38. https://doi.org/10.1097/ SHK.0b013e31827b47bb

14. Huang CT, Lin HH, Ruan SY, Lee MS, Tsai YJ, Yu CJ. Efficacy and adverse events of high-frequency oscillatory ventilation in adult patients with acute respiratory distress syndrome: a meta-analysis. Crit Care 2014;18(3):R102. https://doi.org/10.1186/cc13880

15. Young D, Lamb SE, Shah S, et al.; OSCAR Study Group. Highfrequency oscillation for acute respiratory distress syndrome. N Engl J Med 2013;368(9):806-813. https://doi.org/10.1056/ NEJMoa1215716

16. Putensen $\mathrm{C}$, Wrigge H. Clinical review: biphasic positive airway pressure and airway pressure release ventilation. Crit Care 2004;8(6):492-497. https://doi.org/10.1186/cc2919

17. Güldner A, Braune A, Carvalho N, et al. Higher levels of spontaneous breathing induce lung recruitment and reduce global stress/strain in experimental lung injury. Anesthesiology 2014;120(3):673-682. https:// doi.org/10.1097/ALN.0000000000000124

18. Osiovich HC, Suguihara C, Goldberg RN, Hehre D, Martinez O, Bancalari E. Hemodynamic effects of conventional and high frequency oscillatory ventilation in normal and septic piglets. Biol Neonate 1991;59(4):244-252. https://doi.org/10.1159/000243350

19. Karmrodt J, David M, Yuan S, Markstaller K. Alternative protocol to initiate high-frequency oscillatory ventilation: an experimental study. Crit Care 2006;10(5):R138. https://doi.org/10.1186/cc5052

20. David M, Gervais HW, Karmrodt J, Depta AL, Kempski O, Markstaller K. Effect of a lung recruitment maneuver by high-frequency oscillatory ventilation in experimental acute lung injury on organ blood flow in pigs. Crit Care 2006;10(4):R100. https://doi.org/10.1186/cc4967

21. Estados Unidos Mexicanos. AFÍA. Especificaciones técnicas para la producción, cuidado y uso de animales de laboratorio de la Norma Oficial Mexicana NOM-062-Z00-1999. Diario Oficial de la Federación 6 diciembre, 1999.

22. National Institutes of Health U.S.A. Guía Para el Cuidado y Uso de Los Animales de Laboratorio. Department of Health and Human Services, Public Health Service, National Institutes of Health U.S.A. Edición Mexicana Auspiciada por la Academia Nacional de Medicina. México, D.F. 2002.

23. Herrera-Gutiérrez ME, Seller-Pérez G, Quesada-García G, Granados MM, Domínguez JM, Gómez-Villamandos RJ. Development of a septic shock experimental model oriented at training. Application in the training of depuration techniques in the management of severe sepsis. Med Intensiva 2011;35(2):84-91. https://doi.org/10.1016/j. medin.2010.12.011

24. Koksel O, Kaplan MB, Ozdulger A, et al. Oleic acid-induced lung injury in rats and effects of caffeic acid phenethyl ester. Exp Lung Res 2005;31(5):483-496. https://doi.org/10.1080/01902140590918876

25. Albertini $\mathrm{M}$, Clement $\mathrm{MG}$, Lafortuna $\mathrm{CL}$, et al. Role of poly-(A $\triangle \mathrm{P}$ ribose) synthetase in lipopolysaccharide-induced vascular failure and acute lung injury in pigs. J Crit Care 2000;15(2):73-83. https:// doi.org/10.1053/jcrc.2000.7903

26. Keshari RS, Silasi-Mansat R, Zhu H, et al. Acute lung injury and fibrosis in a baboon Model of Escherichia colisepsis. Am J Respir Cell Mol Biol 2014;50(2):439-450. https://doi.org/10.1165/rcmb.2013-02190C 
Neumol Cir Torax. 2020; 79 (1): 37-49

27. Hii HP, Liao MH, Chen SJ, Wu CC, Shih CC. Distinct patterns of Wnt3a and Wnt5a signaling pathway in the lung from rats with endotoxic shock. PLoS One 2015;10(7):e0134492. https://doi.org/10.1371/ journal.pone.0134492

28. Hancock RE, Scott MG. The role of antimicrobial peptides in animal defenses. Proc Natl Acad Sci U S A 2000;97(16):8856-8861. https:// doi.org/10.1073/pnas.97.16.8856

29. Müller-Leisse C, Klosterhalfen B, Hauptmann S, et al. Computed tomography and histologic results in the early stages of endotoxininjured pig lungs as a model for adult respiratory distress syndrome. Invest Radiol 1993;28(1):39-45. https://doi.org/10.1097/00004424199301000-00012

30. Newman JH, Loyd JE, English JK, Ogletree ML, Fulkerson WJ, Brigham KL. Effects of $100 \%$ oxygen on lung vascular function in awake sheep. J Appl Physiol Respir Environ Exerc Physiol 1983;54(5):1379-1386. https://doi.org/10.1152/jappl.1983.54.5.1379

31. Menzel M, Doppenberg EM, Zauner A, et al. Cerebral oxygenation in patients after severe head injury: monitoring and effects of arterial hyperoxia on cerebral blood flow, metabolism and intracranial pressure. J Neurosurg Anesthesiol 1999;11(4):240-251. https://doi. org/10.1097/00008506-199910000-00003

32. Martin-Flores M, Tseng CT, Robillard SD, et al. Effects of two fractions of inspired oxygen during anesthesia on early postanesthesia oxygenation in healthy dogs. Am J Vet Res 2018;79(2):147-153. https:// doi.org/10.2460/ajvr.79.2.147

33. Emr B, Gatto LA, Roy S, et al. Airway pressure release ventilation prevents ventilator-induced lung injury in normal lungs. JAMA Surg 2013;148(11):1005-1012. https://doi.org/10.1001/jamasurg.2013.3746

34. Guillamet MC, Rhee C, Patterson AJ. Cardiovascular management of septic shock in 2012. Curr Infect Dis Rep 2012;14(5):493-502. https:// doi.org/10.1007/s11908-012-0279-z

35. Briceño I. Sepsis: Etiología, manifestaciones clínicas y diagnóstico. Medicrit 2005;2(9):203-213.

36. Traverse JH, Korvenranta H, Adams EM, Goldthwait DA, Carlo WA. Impairment of hemodynamics with increasing mean airway pressure during high-frequency oscilatory ventilation. Pediatr Res 1988;23(6):628631. https://doi.org/10.1203/00006450-198806000-00020

37. David M, von Bardeleben RS, Weiler N, et al. Cardiac function and haemodynamics during transition to high-frequency oscillatory ventilation. Eur J Anaesthesiol 2004;21(12):944-952. https://doi. org/10.1017/s0265021504000328

38. Smailys A, Mitchell JR, Dolig CJ, Tyberg JV, Belenkie I. High-frequency oscillatory ventilation versus conventional ventilation: hemodynamic effects on lung and heart. Physiol Rep 2014;2(3):e00259. https://doi. org/10.1002/phy2.259

39. Gu XL, Wu GN, Yao YW, Shi DH, Song Y. Is high-frequency oscillatory ventilation more effective and safer than conventional protective ventilation in adult acute respiratory distress syndrome patients? A meta-analysis of randomized controlled trials. Crit Care 2014;18(3):R111. https://doi.org/10.1186/cc13900

40. Zhou Y, Jin X, Lv Y, et al. Early application of airway pressure release ventilation may reduce the duration of mechanical ventilation in acute respiratory distress syndrome. Intensive Care Med 2017;43(11):16481659. https://doi.org/10.1007/s00134-017-4912-z

41. Derdak S, Mehta S, Stewart TE, et al.; Multicenter Oscillatory Ventilation for Acute Respiratory Distress Syndrome Trial (MOAT) Study Investigators. High frecuency oscilatory ventilation for acute respiratory distress syndrome in adults: a randomized, controlled trial. Am J Respir Crit Care Med 2002;166(6):801-808. https://doi. org/10.1164/rccm.2108052

42. Roosens CD, Ama R, Leather HA, et al. Hemodynamic effects of different lung-protective ventilation strategies in closed-chest pigs with normal lungs. Crit Care Med 2006;34(12):2990-2996. https://doi. org/10.1097/01.CCM.0000242758.37427.16

43. Cabrera-Rayo A, Laguna-Hernández G, López-Huerta G, Villagómez-Ortiz A, Méndez-Reyes R, Guzmán-Gómez R. Mecanismos patogénicos en sepsis y choque séptico. Med Int Mex 2008;24(1):38-42.

44. Räsänen J, Downs JB, Stock MC. Cardiovascular effects of conventional positive pressure ventilation and airway pressure release ventilation. Chest 1988;93(5):911-915. https://doi.org/10.1378/ chest.93.5.911

45. Putensen $\mathrm{C}$, Zech S, Wrigge $\mathrm{H}$, et al. Long-term effects of spontaneous breathing during ventilatory support in patients with acute lung injury. Am J Respir Crit Care Med 2001;164(1):43-49. https://doi.org/10.1164/ ajrccm.164.1.2001078

46. Fort $\mathrm{P}$, Farmer $\mathrm{C}$, Westerman J, et al. High-frequency oscillatory ventilation for adult respiratory distress syndrome a pilot study. Crit Care Med 1997;25(6):937-947. https://doi.org/10.1097/00003246199706000-00008

47. Martin-Arsanios D, Barragana AF, Garzón DA, et al. Update in sepsis and septic shock: New definitions and clinical evaluation. Acta Colomb Cuid Intensivo 2017;17(3):158-183. doi: 10.1016/j.acci.2017.03.001

48. Ortiz G, Dueñas C, Garay M, Díaz G. Hypoxic pulmonary vasoconstriction. Acta Colomb Cuid Intensivo 2013;13(4):37-150.

49. Miranda-Ruiz R, Castañón-González JA. Hyperglycemia in critically ill patients: clinical implications for treatment. Cir Ciruj 2004;72(6):517524.

50. James JH, Luchette FA, McCarter FD, Fischer JE. Lactate is an unreliable indicator of tissue hypoxia in injury or sepsis. Lancet 1999;354(9177):505-508. https://doi.org/10.1016/S01406736(98)91132-1

51. Sun H, Huang Y, Yin C, Guo J, Zhao R, Yang X. Lipopolysaccharide markedly changes glucose metabolism and mitochondrial function in the longissimus muscle of pigs. Animal 2016;10(7):1204-1212. https:// doi.org/10.1017/S1751731116000100

52. Nguyen HB, Rivers EP, Knoblich BP, et al. Early lactate clearance is associated with improved outcome in severe sepsis and septic shock. Crit Care Med 2004;32(8):1637-1642. https://doi.org/10.1097/01. ccm.0000132904.35713.a7

53. Kaisers U, Kuhlen R, Keske U, et al. Superimposing positive endexpiratory pressure during partial liquid ventilation in experimental lung injury. Eur Respir J 1998;11(5):1035-1042. https://doi.org/10.118 3/09031936.98.11051035

54. Maxwell RA, Green JM, Waldrop J, et al. A randomized prospective trial of airway pressure release ventilation and low tidal volume ventilation in adult trauma patients with acute respiratory failure. J Trauma 2010;69(3):501-510. https://doi.org/10.1097/TA.0b013e3181e75961

55. Siau C, Stewart TE. Current role of high frequency oscillatory ventilation and airway pressure release ventilation in acute lung injury and acute respiratory distress syndrome. Clin Chest Med 2008;29(2):265-275, vi. https://doi.org/10.1016/j.ccm.2008.02.002

56. Lara-Cruz J, Mendoza-Rodríguez M, López-González A, HuertaValerio R, Sánchez-Domínguez R, Herrera-Morales BE. Ventilación liberadora de presión en vía respiratoria versus ventilación controlada neumoprotectora en falla respiratoria aguda. Rev Asoc Mex Med Crit y Ter 2014;28(2):75-84. 
57. Castañón-González JA, León-Gutiérrez MA, Gallegos-Pérez H, Pech-Quijano J, Martínez-Gutiérrez M, Olvera-Chávez A. Pulmonary mechanics, oxygenation index, and alveolar ventilation in patients with two controlled ventilatory modes. A comparative crossover study. Cir Ciruj 2003;71(5):374-378.

58. Flores-BadillaA, Alvarado-González A. Ventilación mecánica con soporte de presión. Rev Med Costa Rica Centroam 2007;64(581):223-228.

59. Albert RK. The role of ventilation-induced surfactant dysfunction and atelectasis in causing acute respiratory distress syndrome. Am
J Respir Crit Care Med 2012;185(7):702-708. https://doi.org/10.1164/ rccm.201109-1667PP

60. Eastman A, Holland D, Higgins J, et al. High-frequency percussive ventilation improves oxygenation in trauma patients with acute respiratory distress syndrome: a retrospective review. Am J Surg 2006;192(2):191-195. https://doi.org/10.1016/j.amjsurg.2006.01.021

Conflicto de intereses: Los autores declaran no tener conflicto de intereses. 